\title{
On the Equivalence of Experimental B(E2) Values Determined by Various Techniques
}

\author{
M. Birch ${ }^{\mathrm{a}}$, B. Pritychenko ${ }^{\mathrm{b}, *}$, B. Singh ${ }^{\mathrm{a}}$ \\ ${ }^{a}$ Department of Physics $\&$ Astronomy, McMaster University, Hamilton, \\ Ontario L8S 4M1, Canada \\ ${ }^{b}$ National Nuclear Data Center, Brookhaven National Laboratory, \\ Upton, NY 11973-5000, USA
}

\begin{abstract}
We establish the equivalence of the various techniques for measuring $\mathrm{B}(\mathrm{E} 2)$ values using a statistical analysis. Data used in this work come from the recent compilation by B. Pritychenko et al., At. Data Nucl. Data Tables 107 (2016). We consider only those nuclei for which the $\mathrm{B}(\mathrm{E} 2)$ values were measured by at least two different methods, with each method being independently performed at least twice. Our results indicate that most prevalent methods of measuring $\mathrm{B}(\mathrm{E} 2)$ values are equivalent, with some weak evidence that Doppler-shift attenuation method (DSAM) measurements may differ from Coulomb excitation (CE) and nuclear resonance fluorescence (NRF) measurements. However, such an evidence appears to arise from discrepant DSAM measurements of the lifetimes for ${ }^{60} \mathrm{Ni}$ and some Sn nuclei rather than a systematic deviation in the method itself.
\end{abstract}

Keywords: Reduced Transition Probabilities, Nuclear Data Analysis, Coulomb Excitation

PACS: 23.20.-g, 29.85.-c, 25.70.De

\section{Introduction}

Reduced transition probabilities, the $\mathrm{B}(\mathrm{E} 2)$ values for transitions from the first excited $2^{+}$to the ground states in even-even nuclei are fundamentally important quantities in nuclear physics for determining the collectivity

\footnotetext{
*Corresponding author

Email address: pritychenko@bnl.gov (B. Pritychenko)
} 
in nuclei. As such, experimental efforts to measure B(E2) values have been going on for the past sixty years and many different techniques have been developed for this purpose. The two most common methods are Coulomb excitation (CE) and Doppler-shift attenuation (DSAM). A recent CE study of the B(E2) values for Sn nuclei [1] compared their measurements with previous works and revealed an apparent systematic disagreement with a set of DSAM measurements [2]. Without doubting the standard formulations relating $\mathrm{B}(\mathrm{E} 2)$ and lifetime, this does raise the question that, given the different sets of systematic errors associated with different techniques, are the results significantly impacted such that the methods may or may not be considered equivalent. To the best of our knowledge, this question has never been addressed comprehensively across the entire chart of nuclides while comparing different methods, although, limited comparisons have been published between CE experiments at different energies [3, 4].

Our newly published compilation and evaluation of experimental B(E2) values [5] for the first $2^{+}$states in the even-even nuclei, an extensive update of the previous work by Raman et al. [6], provides an opportunity to adequately answer the question of whether systematic differences exist between different methods of determining B(E2) values. In particular, we focus here on the most common experimental techniques for measuring $\mathrm{B}(\mathrm{E} 2)$ values, namely: Doppler-shift attenuation method (DSAM), recoil distance Dopplershift (RDDS), delayed coincidences (DC), Coulomb excitation (CE), nuclear resonance fluorescence (NRF) and the weakly model dependent method of electron scattering $\left(e, e^{\prime}\right)$. Note that DSAM includes also "transmission" Doppler-shift attenuation in which particles are still travelling through the medium when gamma-rays are detected. In making comparisons between methods, using our compiled data, we have selected only those nuclei which have had their B(E2) values measured by at least two different methods, with each method being independently employed by different laboratories at least twice. This requirement implies that we can always take an average when determining the result from a particular method, reducing the likelihood of an outlying measurement having a large influence on our conclusions. As a result, of the 447 nuclei which were included in our compilation, we can only use measurements on 100 of those nuclei. Table A1 lists these nuclei and their $\mathrm{B}(\mathrm{E} 2)$ values as determined by the different experimental methods. 


\section{Analysis}

Our statistical analysis is performed as follows: let $x \pm \delta x$ and $y \pm \delta y$ be two measurements of the $\mathrm{B}(\mathrm{E} 2)$ value of the same nucleus determined by different methods. Note that since we insist that each experimental method be performed at least twice on each isotope, $x$ and $y$ are each an average of at least two independent measurements. We define the normalized difference between the two results, $x \pm \delta x$ and $y \pm \delta y$, as

$$
z=\frac{x-y}{\sqrt{\delta x^{2}+\delta y^{2}}} .
$$

Assume that $x$ was sampled from a random variable, $X$, which is normally distributed such that $\langle X\rangle$ is the "true value" of the $\mathrm{B}(\mathrm{E} 2)$ value for the nucleus and $\operatorname{Var}(X)=\delta x^{2}$. Similarly, assume $y$ was sampled from a normally distributed random variable, $Y$, such that $\operatorname{Var}(Y)=\delta y^{2}$. The two experimental procedures will be equivalent for this nucleus if $\langle X\rangle=\langle Y\rangle$. Under these assumptions, $z$ will be sampled from a normal distribution with mean given by

$$
\langle Z\rangle=\frac{\langle X\rangle-\langle Y\rangle}{\sqrt{\delta x^{2}+\delta y^{2}}}
$$

and unit variance. Clearly, if the two experimental methods are equivalent, then $\langle Z\rangle=0$, independent of which nucleus is being considered. Also notice that since $z$ is dimensionless and $\operatorname{Var}(Z)=1$, we can interpret $z$ as being the difference between $x$ and $y$ in units of standard deviations of $(X-Y)$. Now consider two sets of B(E2) measurements, $\left\{x_{i} \pm \delta x_{i}\right\}_{i=1}^{N}$ and $\left\{y_{i} \pm \delta y_{i}\right\}_{i=1}^{N}$. Each $x_{i}$ is determined by one experimental method, while each $y_{i}$ is determined by another. The two sets are paired such that $x_{i}$ and $y_{i}$ are measurements on the same nucleus and hence for each such pair we can calculate $z_{i}$ according to equation (1). Under the hypothesis that the two methods are equivalent, $\left\{z_{i}\right\}_{i=1}^{N}$ is a sample of size $N$ from a standard normal distribution (i.e. one with zero mean and unit variance). However, as we shall show later, the actual distribution of $\left\{z_{i}\right\}_{i=1}^{N}$ we observe has some degree of asymmetry. This could arise due to underestimated uncertainties since that would artificially increase the resulting values of $z$. We circumvent this problem phenomenologically by postulating (and later provide evidence using the real data) that the distribution of $\left\{z_{i}\right\}_{i=1}^{N}$ is in fact an asymmetric 
normal distribution, with probability density function given by

$$
f(z ; m, a, b)=\left\{\begin{array}{l}
\sqrt{\frac{2}{\pi(a+b)^{2}}} \exp \left(-\frac{1}{2} \frac{(z-m)^{2}}{b^{2}}\right), z \leq m \\
\sqrt{\frac{2}{\pi(a+b)^{2}}} \exp \left(-\frac{1}{2} \frac{(z-m)^{2}}{a^{2}}\right), z>m
\end{array},\right.
$$

where $m$ is the mode (most probable value) of the distribution, and $a, b$ give the upper and lower standard deviations, respectively. By fitting the values of $m, a$ and $b$ to the distribution of $\left\{z_{i}\right\}_{i=1}^{N}$, we can compute the probability that methods $x$ and $y$ will differ by $n$ standard deviations as (recall the interpretation of $z$ above)

$$
P_{n}=\operatorname{Pr}(|z|>n)=\int_{-\infty}^{-n} f(z ; m, a, b) d z+\int_{n}^{\infty} f(z ; m, a, b) d z,
$$

the average difference as

$$
\mu_{d}=\int_{-\infty}^{\infty} z f(z ; m, a, b) d z=m+\sqrt{\frac{2}{\pi}}(a-b),
$$

and the most probable deviation is simply $m$. Each of these quantities provides a different measure of difference between methods $x$ and $y$. Using these measures we define three different criteria for determining that two methods are not equivalent:

(i) $P_{1}>0.5$, i.e. it is more probable that $(x-y)$ differs from zero by at least one standard deviation than not;

(ii) $\left|\mu_{d}\right|>1$, i.e. on average $(x-y)$ differs from zero by at least one standard deviation;

(iii) $|m|>1$, i.e. the most probable value of $(x-y)$ differs from zero by more than one standard deviation.

Criteria (ii) and (iii) also give information regarding the direction of the deviation, e.g. if $\mu_{d}<-1$ then not only can we say that $x$ is not equivalent to $y$, but that it is on average less than $y$. 


\section{Results}

Let us consider a particular example to illustrate this analysis in detail. The two most common methods for determining $\mathrm{B}(\mathrm{E} 2)$ values are $\mathrm{CE}$ and DSAM, so let us compare those methods (i.e. choose $x=\mathrm{CE}$ and $y=$ DSAM). There are 43 nuclei which have at least two independent DSAM and two independent CE measurements. We can compute the normalized differences between the two methods, $z_{i}$, as defined above for these 43 cases. To show that these data are not normally distributed, we performed a ShapiroWilk test [7]. This test uses a statistic denoted $W$ which is close to unity for normally distributed data and close to zero otherwise. For this dataset, we found that $W=0.928$, which gives a p-value of 0.0096 , i.e. the probability of obtaining that value of $W$, assuming that the data are normally distributed, is less than $1 \%$. Therefore, it is statistically significant that $\left\{z_{i}\right\}_{i=1}^{43}$ are not normally distributed. Instead we can fit an asymmetric normal distribution to $\left\{z_{i}\right\}_{i=1}^{43}$ and obtain $m=-0.218, a=1.93, b=1.12$. Using Pearson's

$\chi^{2}$ test for goodness of fit, $\chi^{2} /(N-3)=0.756$, which is smaller than the critical $\chi^{2}$ for rejection at $99 \%$ confidence level, indicating an acceptable fit to the data. Hence we have shown, as claimed above, that $z_{i}$ is not normally distributed, but is well-modelled as being distributed according to an asymmetric normal distribution. A histogram of $\left\{z_{i}\right\}_{i=1}^{43}$ together with the asymmetric normal fit is shown in Fig. 1a. Using this fit we can calculate the various quantities defined above to test for equivalence between $\mathrm{CE}$ and DSAM: $P_{1}=0.513, \mu_{d}=-0.428$ and $m=-0.218$. Therefore, we would conclude that DSAM and CE are equivalent methods using criteria (ii) and (iii), however they are not equivalent by criterion (i). This discrepancy is further discussed in Section 4. Fig. 1a could be misleading in the sense that the remaining distribution, after removing the outlying point, appears symmetric and so one might concluded that using an asymmetric distribution in this analysis is not actually necessary. To clarify this point, Fig. 1b shows a histogram of the $z$ values for the $\mathrm{CE} /\left(e, e^{\prime}\right)$ pair. In this case $P_{1}=0.256$, $\mu_{d}=0.362$ and $m=-0.667$, hence the two methods are equivalent according to all three criteria. However, we see that the distribution is still quite asymmetric. The histograms of $z$ values for $\mathrm{CE}$ with the remaining methods (DC, RDDS, NRF) are given in Appendix B also show asymmetric distributions.

In order to systematically establish the equivalence of each method to one another, we performed this analysis for each pair of methods and did not find any pairs which satisfied criteria (ii) or (iii), although a DSAM/CE 


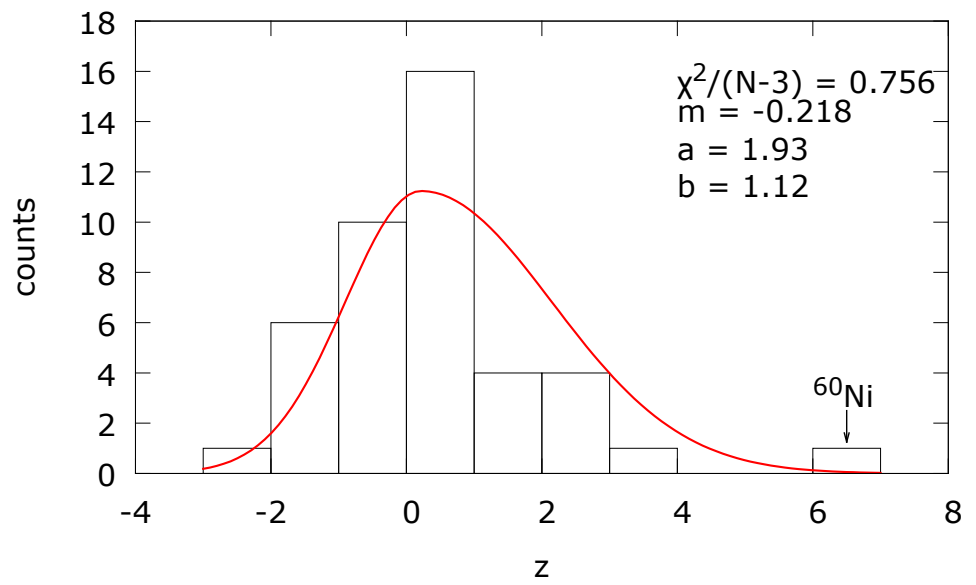

(a)

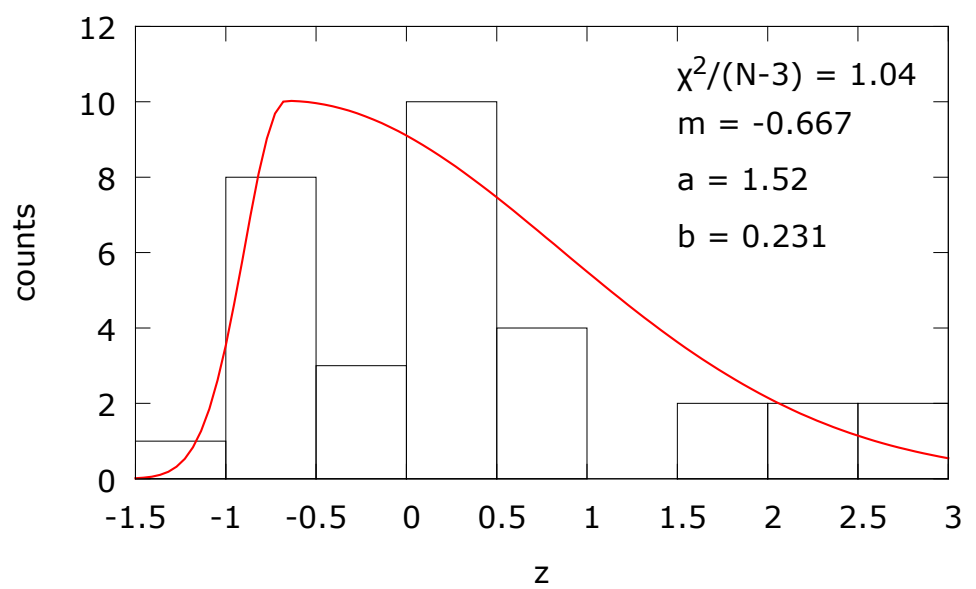

(b)

Figure 1: (a) Histograms of the $z$ values computed for the CE/DSAM method pair, together with the asymmetric normal distribution fit. The ${ }^{60} \mathrm{Ni}$ outlier in this dataset is also indicated. (b) Same as (a), but for the $\mathrm{CE} /\left(e, e^{\prime}\right)$ pair. 


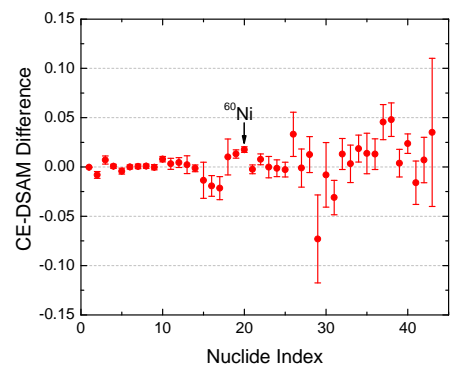

(a)

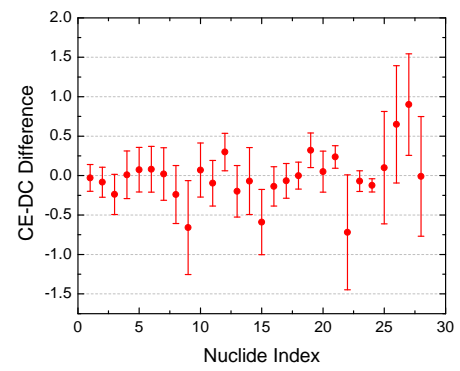

(c)

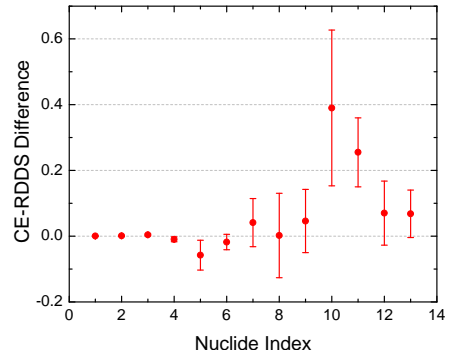

(b)

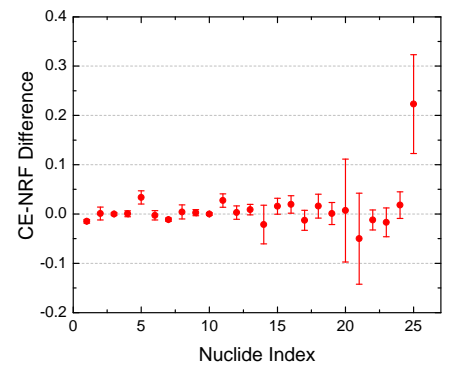

(d)

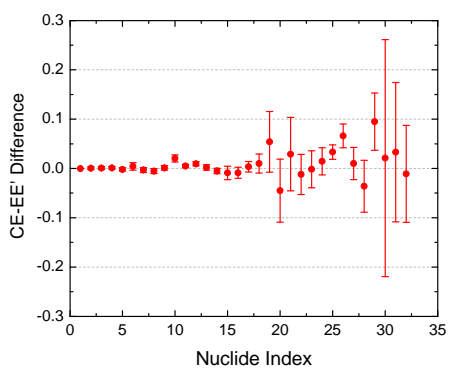

(e)

Figure 2: Difference between $\mathrm{B}(\mathrm{E} 2)$ values determined by $\mathrm{CE}$ and each other method: (a), (b), (c), (d) (e) compare with DSAM, RDDS, DC, NRF and (e,e' $)$ respectively. The $\mathrm{x}$-axis in each plot shows the index associated with the nuclide for that pairing of methods. These indices are essentially arbitrary and differ between the different plots, but increasing index always corresponds to increasing mass number. See Table C1 to see which nuclide corresponds to each index. 
Table 1: Comparison between $\mathrm{CE}$ and five other methods using each of the metrics outlined in equations (3), (4) and (5) and used to define non-equivalence criteria (i)-(iii). The quantity $\chi^{2} /(N-3)$ gives the reduced $\chi^{2}$ values for the fit of an asymmetric normal distribution to the calculated $\left\{z_{i}\right\}_{i=1}^{N}$, establishing the goodness of fit.

\begin{tabular}{ccccc}
\hline \hline Method & $\chi^{2} /(N-3)$ & $P_{1}$ & $\mu_{d}$ & $m$ \\
\hline DSAM $^{*}$ & 0.756 & 0.513 & 0.428 & -0.218 \\
RDDS & 0.491 & 0.423 & 0.484 & 0.697 \\
DC & 0.533 & 0.229 & 0.0156 & -0.220 \\
NRF & 2.51 & 0.444 & -0.236 & 0.116 \\
$e, e^{\prime}$ & 1.04 & 0.256 & 0.362 & -0.667 \\
\hline \hline
\end{tabular}

* If the ${ }^{60} \mathrm{Ni}$ DSAM measurements are excluded then $P_{1}=0.469, \mu_{d}=0.264, m=0.0944$.

and DSAM/NRF did satisfy (i). However, in both of these cases neither of the other two criteria are also satisfied. This gives some evidence that there may be problems with some of DSAM measurements, but not necessarily anything systematically wrong with the method itself. Indeed, if the DSAM measurements for ${ }^{60} \mathrm{Ni}$ are excluded then the discrepancy with $\mathrm{CE}$ and NRF no longer exists. This is discussed in detail in Section 4. In this global comparison, we also do not have any evidence for a systematic deviation of $\mathrm{B}(\mathrm{E} 2)$ values in Sn nuclides as suggested in the recent study [1].

Since CE is the most commonly used method, and presumably it suffers less from systematic errors such as stopping powers in DSAM method, detailed results for comparing CE with several other methods are shown in Table 1. Table 1 also further illustrates the asymmetry of the $z$-value distributions. For a symmetric distribution (i.e. usual normal distribution) $\mu_{d}=m$, yet in the table we see the mean and mode differ significantly in all cases. The comparison between $\mathrm{CE}$ and each of the other methods can also be seen graphically in Fig. 2 where the difference (not normalized to the size of the error bars, i.e. $(x-y)$ and not $z)$ between the $\mathrm{B}(\mathrm{E} 2)$ values measured by $\mathrm{CE}$ and each of the other methods are plotted.

\section{Discussion and Conclusion}

The results of Table 1 overall indicate that each method used to measure $\mathrm{B}(\mathrm{E} 2)$ values is equivalent to $\mathrm{CE}$, however some points are worth further discussion. All the $\chi^{2} /(N-3)$ values are smaller than the critical $\chi^{2}$ values 
for rejection at $99 \%$ confidence except $\mathrm{NRF}$, i.e. only the CE/NRF normalized differences are not well-modelled by an asymmetric normal distribution. However, a visual inspection of Fig. $2 d$ reveals that, even if the numeric values of the parameters for NRF in Table 1 are not meaningful, the conclusion of equivalence is still supported. Indeed, about $75 \%$ of the $\mathrm{CE} / \mathrm{NRF}$ differences lie within one standard deviation of zero.

The only indication of non-equivalence in Table 1 is $P_{1}>0.5$ for CE/DSAM. Although not listed, it is also true that $P_{1}>0.5$ for NRF/DSAM as well. This could indicated a systematic problem with DSAM measurements, however the reason for these discrepancies can be attributed to measurements on a single nucleus, ${ }^{60} \mathrm{Ni}$. As can be seen in the histogram of Fig. 1a a single $z$ value is separated from the bulk of the distribution by at least a full unit (standard deviation). This data point is also indicated in Fig. 2a and one can see that indeed the error bars are very small compared to its deviation from zero (hence the large value of $z$ for this measurement). This disagreement between CE and DSAM measurements was also noted in [8], although very good agreement was found for ${ }^{62} \mathrm{Ni}$. Further evidence against the DSAM lifetime measurements of ${ }^{60} \mathrm{Ni}$ is the fact that they are in mutual disagreement with one another. The more recent measurements give $\sim 1.3$ ps $[9,10]$ and earlier measurements $\sim 1$ ps $[11,12]$. This could be due to problems with one or more of the DSAM measurements of this nucleus as a result of poorly determined stopping powers, improperly implemented inverse kinematics during data analysis or ignored possible angular correlations. In any case, it appears that the ${ }^{60} \mathrm{Ni}$ DSAM measurements are an isolated problem rather than evidence of a broad systematic issue with the method. By excluding these measurements from the analysis, there is no longer significant evidence that DSAM disagrees with CE or NRF.

In conclusion, based on the analyses performed on the most recently compiled $\mathrm{B}(\mathrm{E} 2)$ experimental data [5], we conclude that the most commonly methods used in the measurement of $\mathrm{B}(\mathrm{E} 2)$ values are equivalent. There is some evidence to suggest that there is disagreement between DSAM and CE as well as DSAM and NRF methods, however these appear to arise from discrepant DSAM measurements in the lifetime of ${ }^{60} \mathrm{Ni}$ and not from a systematic deficiency in the method itself. Similarly, we do not find evidence for systematic deviation between DSAM and CE results for Sn nuclei, when all the available experimental data are considered. It is possible that DSAM results reported in [2] suffer from some experimental difficulties. 


\section{Acknowledgments}

We are indebted to Dr. M. Herman (BNL) for support of this project. The authors gratefully acknowledge J.M. Allmond (ORNL) for providing his latest results and fruitful discussions. Work at Brookhaven was funded by the Office of Nuclear Physics, Office of Science of the U.S. Department of Energy, under Contract No. DE-AC02-98CH10886 with Brookhaven Science Associates, LC. Work at McMaster University was partially supported by the Office of Science of the U.S. Department of Energy. 


\section{Appendix A. Table of Data}

Table A1: B(E2) recalculated weighted averages for DSAM, RDDS, DC, CE, NRF and $e, e^{\prime}$ experimental methods using the relevant compiled data in Table 1 of 2016 compilation $[5]$.

\begin{tabular}{|c|c|c|c|c|c|}
\hline \multirow{2}{*}{ Nuclei } & \multicolumn{5}{|c|}{$\overline{\mathrm{B}(\mathbf{E} 2) \uparrow\left(e^{2} b^{2}\right)}$} \\
\hline & DSAM & RDDS & $\mathrm{CE}$ & NRF & $e, e^{\prime}$ \\
\hline${ }^{12} \mathrm{C}$ & $0.00400(20)$ & & & $0.00334(71)$ & $0.00399(33)$ \\
\hline${ }^{16} \mathrm{O}$ & & & & $0.00371(32)$ & $0.00413(36)$ \\
\hline${ }^{18} \mathrm{O}$ & $0.00458(24)$ & $0.00405(20)$ & $0.00429(15)$ & & $0.00449(22)$ \\
\hline${ }^{18} \mathrm{Ne}$ & $0.0241(22)$ & & $0.0160(26)$ & & \\
\hline${ }^{20} \mathrm{Ne}$ & $0.0299(28)$ & & $0.0369(30)$ & & \\
\hline${ }^{22} \mathrm{Ne}$ & $0.0230(15)$ & $0.0229(11)$ & $0.0237(14)$ & & $0.0232(22)$ \\
\hline${ }^{24} \mathrm{Mg}$ & $0.0472(23)$ & $0.0391(19)$ & $0.0430(20)$ & $0.0578(32)$ & $0.0421(23)$ \\
\hline${ }^{26} \mathrm{Mg}$ & $0.0309(15)$ & & $0.0309(15)$ & $0.030(13)$ & $0.0298(21)$ \\
\hline${ }^{28} \mathrm{Si}$ & $0.0323(16)$ & & $0.0329(17)$ & $0.0330(27)$ & $0.0348(31)$ \\
\hline${ }^{32} \mathrm{~S}$ & $0.0293(14)$ & & $0.0301(16)$ & $0.0296(58)$ & $0.0259(74)$ \\
\hline${ }^{34} \mathrm{~S}$ & $0.0210(12)$ & & $0.0207(24)$ & & \\
\hline${ }^{36} \mathrm{Ar}$ & $0.0219(16)$ & & $0.0298(23)$ & & \\
\hline${ }^{40} \mathrm{Ar}$ & $0.0327(24)$ & & $0.0359(50)$ & & \\
\hline${ }^{40} \mathrm{Ca}$ & $0.0093(10)$ & & & $0.0103(11)$ & $0.00703(82)$ \\
\hline${ }^{42} \mathrm{Ca}$ & $0.0334(24)$ & & & $0.060(12)$ & $0.0367(49)$ \\
\hline${ }^{44} \mathrm{Ca}$ & $0.0440(36)$ & & $0.0485(35)$ & & $0.0517(35)$ \\
\hline${ }^{48} \mathrm{Ca}$ & $0.0085(13)$ & & & & $0.00826(50)$ \\
\hline${ }^{46} \mathrm{Ti}$ & & $0.0995(48)$ & $0.0898(60)$ & $0.056(12)$ & \\
\hline${ }^{48} \mathrm{Ti}$ & $0.0626(55)$ & & $0.0649(72)$ & $0.0676(60)$ & \\
\hline${ }^{50} \mathrm{Ti}$ & $0.0281(19)$ & & $0.0267(29)$ & & \\
\hline${ }^{50} \mathrm{Cr}$ & $0.118(18)$ & & $0.1045(32)$ & & \\
\hline${ }^{52} \mathrm{Cr}$ & $0.077(10)$ & & $0.0573(31)$ & $0.0687(13)$ & $0.0627(39)$ \\
\hline${ }^{54} \mathrm{Fe}$ & $0.077(11)$ & & $0.0551(38)$ & & $0.0539(24)$ \\
\hline${ }^{56} \mathrm{Fe}$ & $0.088(18)$ & & $0.0981(26)$ & $0.094(14)$ & $0.0777(67)$ \\
\hline${ }^{58} \mathrm{Ni}$ & $0.0560(37)$ & & $0.0692(20)$ & $0.0665(59)$ & $0.0643(25)$ \\
\hline${ }^{60} \mathrm{Ni}$ & $0.0750(21)$ & & $0.0926(20)$ & $0.0927(20)$ & $0.0832(37)$ \\
\hline${ }^{62} \mathrm{Ni}$ & $0.0908(34)$ & & $0.0884(30)$ & & $0.0862(46)$ \\
\hline${ }^{64} \mathrm{Ni}$ & $0.0590(40)$ & & $0.0668(38)$ & & $0.0720(41)$ \\
\hline${ }^{64} \mathrm{Zn}$ & $0.1512(42)$ & & $0.151(10)$ & $0.1237(93)$ & $0.1601(90)$ \\
\hline
\end{tabular}




\begin{tabular}{|c|c|c|c|c|c|c|}
\hline \multirow{2}{*}{ Nuclei } & \multicolumn{6}{|c|}{$\overline{\mathbf{B}(\mathbf{E} 2) \uparrow\left(e^{2} b^{2}\right)}$} \\
\hline & DSAM & RDDS & $\mathrm{DC}$ & $\mathrm{CE}$ & NRF & $e, e^{\prime}$ \\
\hline${ }^{66} \mathrm{Zn}$ & $0.1379(29)$ & & & $0.1366(80)$ & $0.134(11)$ & $0.1454(80)$ \\
\hline${ }^{68} \mathrm{Zn}$ & $0.1206(26)$ & & & $0.1179(70)$ & $0.1091(81)$ & $0.1145(80)$ \\
\hline${ }^{70} \mathrm{Zn}$ & $0.1429(80)$ & & & $0.176(21)$ & & \\
\hline${ }^{70} \mathrm{Ge}$ & $0.179(19)$ & & & $0.1779(35)$ & & \\
\hline${ }^{72} \mathrm{Ge}$ & $0.196(18)$ & & & $0.2086(30)$ & $0.230(39)$ & \\
\hline${ }^{72} \mathrm{Se}$ & $0.183(20)$ & $0.190(15)$ & & & & \\
\hline${ }^{78} \mathrm{Kr}$ & $0.674(33)$ & $0.659(34)$ & & $0.601(30)$ & & \\
\hline${ }^{80} \mathrm{Kr}$ & $0.396(26)$ & & & $0.388(20)$ & & \\
\hline${ }^{86} \mathrm{Sr}$ & $0.1400(70)$ & & & $0.109(16)$ & & \\
\hline${ }^{88} \mathrm{Sr}$ & $0.0909(47)$ & & & $0.104(15)$ & $0.0882(59)$ & $0.094(12)$ \\
\hline${ }^{90} \mathrm{Zr}$ & $0.086(13)$ & & & & & $0.057(10)$ \\
\hline${ }^{92} \mathrm{Mo}$ & $0.102(18)$ & & & $0.1052(60)$ & & \\
\hline${ }^{94} \mathrm{Mo}$ & $0.1959(95)$ & & & $0.2145(98)$ & & \\
\hline${ }^{96} \mathrm{Mo}$ & $0.269(15)$ & & & $0.283(14)$ & & \\
\hline${ }^{96} \mathrm{Ru}$ & $0.231(10)$ & & & $0.244(12)$ & & \\
\hline${ }^{98} \mathrm{Ru}$ & & $0.413(14)$ & & $0.395(19)$ & & \\
\hline${ }^{106} \mathrm{Pd}$ & & & & $0.662(37)$ & & $0.608(49)$ \\
\hline${ }^{108} \mathrm{Pd}$ & & & & $0.762(50)$ & & $0.807(40)$ \\
\hline${ }^{110} \mathrm{Pd}$ & & $0.838(42)$ & & $0.879(60)$ & & $0.850(44)$ \\
\hline${ }^{110} \mathrm{Cd}$ & & & & $0.438(21)$ & & $0.450(35)$ \\
\hline${ }^{114} \mathrm{Cd}$ & & & & $0.536(25)$ & & $0.538(28)$ \\
\hline${ }^{112} \mathrm{Sn}$ & $0.195(14)$ & & & $0.241(11)$ & & \\
\hline${ }^{114} \mathrm{Sn}$ & $0.185(12)$ & & & $0.233(12)$ & & \\
\hline${ }^{116} \mathrm{Sn}$ & & & & $0.2135(50)$ & $0.194(17)$ & $0.199(27)$ \\
\hline${ }^{118} \mathrm{Sn}$ & & & & $0.2052(40)$ & $0.218(20)$ & $0.172(14)$ \\
\hline${ }^{120} \mathrm{Sn}$ & $0.1982(98)$ & & & $0.202(10)$ & $0.186(22)$ & $0.136(22)$ \\
\hline${ }^{124} \mathrm{Sn}$ & $0.1414(90)$ & & & $0.1651(41)$ & $0.164(22)$ & \\
\hline${ }^{122} \mathrm{Te}$ & & & & $0.647(30)$ & $0.64(10)$ & \\
\hline${ }^{124} \mathrm{Te}$ & & & & $0.566(28)$ & $0.616(88)$ & \\
\hline${ }^{124} \mathrm{Xe}$ & & $0.978(66)$ & & $0.98(11)$ & & \\
\hline${ }^{130} \mathrm{Ba}$ & & $1.111(55)$ & & $1.157(79)$ & & \\
\hline${ }^{138} \mathrm{Ba}$ & & & & $0.229(11)$ & $0.241(17)$ & \\
\hline${ }^{144} \mathrm{Ba}$ & & $1.02(18)$ & $1.020(55)$ & & & \\
\hline${ }^{140} \mathrm{Ce}$ & $0.307(16)$ & & & $0.291(15)$ & $0.308(25)$ & \\
\hline
\end{tabular}




\begin{tabular}{|c|c|c|c|c|c|c|}
\hline \multirow{2}{*}{ Nuclei } & \multicolumn{6}{|c|}{$\overline{\mathbf{B}(\mathbf{E} 2) \uparrow\left(e^{2} b^{2}\right)}$} \\
\hline & DSAM & RDDS & $\mathrm{DC}$ & $\mathrm{CE}$ & NRF & $e, e^{\prime}$ \\
\hline${ }^{142} \mathrm{Ce}$ & & & & $0.467(23)$ & & $0.457(23)$ \\
\hline${ }^{142} \mathrm{Nd}$ & $0.265(13)$ & & & $0.272(19)$ & $0.254(19)$ & $0.308(49)$ \\
\hline${ }^{146} \mathrm{Nd}$ & & & & $0.750(38)$ & & $0.655(44)$ \\
\hline${ }^{144} \mathrm{Sm}$ & $0.228(74)$ & & & $0.263(13)$ & & \\
\hline${ }^{152} \mathrm{Sm}$ & & & $3.4613(23)$ & $3.43(17)$ & & $3.41(17)$ \\
\hline${ }^{154} \mathrm{Gd}$ & & & $3.874(16)$ & $3.79(19)$ & & \\
\hline${ }^{156} \mathrm{Gd}$ & & & $4.77(11)$ & $4.53(23)$ & & \\
\hline${ }^{158} \mathrm{Gd}$ & & & $5.08(17)$ & $5.09(25)$ & & \\
\hline${ }^{160} \mathrm{Gd}$ & & & $5.21(11)$ & $5.28(26)$ & & \\
\hline${ }^{156} \mathrm{Dy}$ & & & $3.66(22)$ & $3.74(19)$ & & \\
\hline${ }^{158}$ Dy & & & $4.65(24)$ & $4.67(23)$ & & \\
\hline${ }^{162} \mathrm{Dy}$ & & & $5.27(26)$ & $5.03(26)$ & & \\
\hline${ }^{162} \mathrm{Er}$ & & & $5.61(54)$ & $4.95(25)$ & & \\
\hline${ }^{164} \mathrm{Er}$ & & & $5.25(21)$ & $5.32(27)$ & & \\
\hline${ }^{166} \mathrm{Er}$ & & & $5.78(16)$ & $5.68(24)$ & & \\
\hline${ }^{168} \mathrm{Er}$ & & & $5.681(59)$ & $5.98(23)$ & & \\
\hline${ }^{172} \mathrm{Yb}$ & & & $6.16(15)$ & $5.96(29)$ & & \\
\hline${ }^{174} \mathrm{Yb}$ & & & $5.91(31)$ & $5.84(29)$ & & \\
\hline${ }^{174} \mathrm{Hf}$ & & & $5.89(22)$ & $5.30(35)$ & & \\
\hline${ }^{178} \mathrm{Hf}$ & & & $4.797(94)$ & $4.66(23)$ & & \\
\hline${ }^{180} \mathrm{Hf}$ & & & $4.6471(30)$ & $4.58(22)$ & & \\
\hline${ }^{182} \mathrm{~W}$ & & & $4.091(61)$ & $4.09(16)$ & & \\
\hline${ }^{184} \mathrm{~W}$ & & & $3.57(11)$ & $3.89(19)$ & & \\
\hline${ }^{186} \mathrm{Os}$ & & & $3.060(72)$ & $3.11(25)$ & & \\
\hline${ }^{188} \mathrm{Os}$ & & & $2.449(59)$ & $2.69(13)$ & & \\
\hline${ }^{190} \mathrm{Os}$ & & $1.98(21)$ & $3.09(72)$ & $2.37(11)$ & & \\
\hline${ }^{192} \mathrm{Os}$ & & & & $2.04(10)$ & & $2.01(10)$ \\
\hline${ }^{192} \mathrm{Pt}$ & & & $2.002(95)$ & $1.931(90)$ & & \\
\hline${ }^{194} \mathrm{Pt}$ & & $1.428(68)$ & & $1.683(80)$ & & \\
\hline${ }^{196} \mathrm{Pt}$ & & $1.348(70)$ & & $1.418(68)$ & & $1.429(71)$ \\
\hline${ }^{198} \mathrm{Pt}$ & & $1.030(52)$ & & $1.098(50)$ & & \\
\hline${ }^{198} \mathrm{Hg}$ & & & $1.084(83)$ & $0.9600(68)$ & $0.74(10)$ & \\
\hline${ }^{208} \mathrm{~Pb}$ & & & & & $0.263(18)$ & $0.312(16)$ \\
\hline${ }^{230} \mathrm{Th}$ & & & $8.12(21)$ & $8.22(68)$ & & \\
\hline
\end{tabular}




\begin{tabular}{|c|c|c|c|c|c|c|}
\hline \multirow{2}{*}{ Nuclei } & \multicolumn{6}{|c|}{$\overline{\mathbf{B}(\mathbf{E 2}) \uparrow\left(e^{2} b^{2}\right)}$} \\
\hline & DSAM & RDDS & $\mathrm{DC}$ & $\mathrm{CE}$ & NRF & $e, e^{\prime}$ \\
\hline${ }^{234} \mathrm{U}$ & & & $9.92(50)$ & $10.57(55)$ & & \\
\hline${ }^{236} \mathrm{U}$ & & & $10.78(28)$ & $11.68(58)$ & & \\
\hline${ }^{240} \mathrm{Pu}$ & & & $13.12(39)$ & $13.11(65)$ & & \\
\hline
\end{tabular}




\section{Appendix B. Additional $z$-Value Histograms}

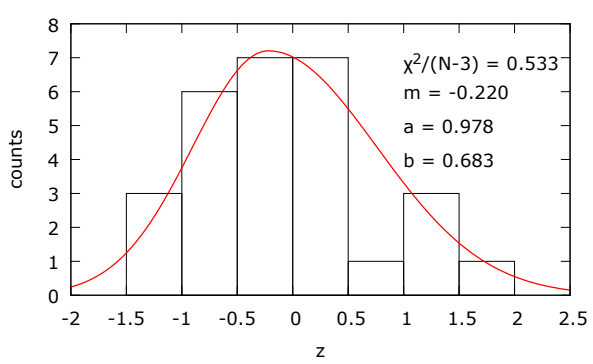

(a)

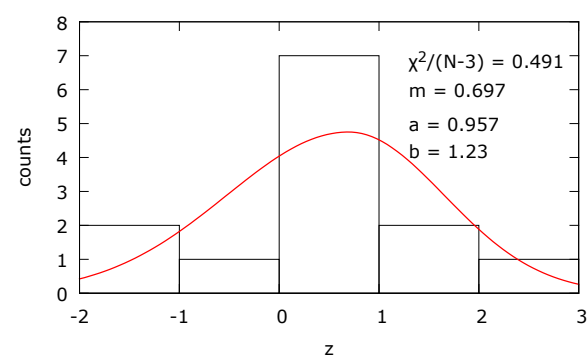

(b)

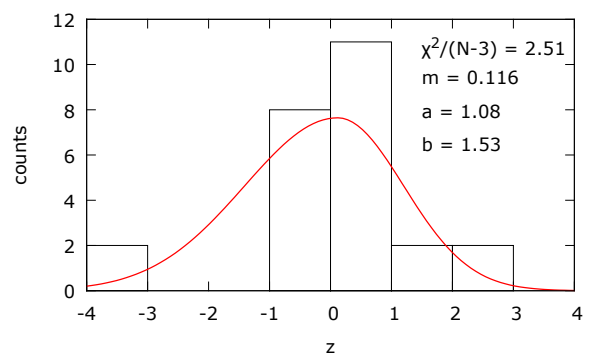

(c)

Figure B1: (a) Histograms of the $z$ values computed for the CE/DC method pair, together with the asymmetric normal distribution fit. (b) Same as (a), but for the CE/RDDS pair. (c) Same as (a), but for the CE/NRF pair. 
Appendix C. Nuclide Indices 
Table C1: List of nuclides corresponding to the indices given on the x-axes of the plots in Fig. 2.

\begin{tabular}{|c|c|c|c|c|c|c|c|}
\hline Index & Fig. $2 \mathrm{a}$ & Fig. 2b & Fig. 2c & Fig. 2d & Fig. 2e & Index & Fig. 2a \\
\hline 1 & ${ }^{18} \mathrm{O}$ & ${ }^{18} \mathrm{O}$ & ${ }^{152} \mathrm{Sm}$ & ${ }^{24} \mathrm{Mg}$ & ${ }^{18} \mathrm{O}$ & 33 & ${ }^{92} \mathrm{Mo}$ \\
\hline 2 & ${ }^{18} \mathrm{Ne}$ & ${ }^{22} \mathrm{Ne}$ & ${ }^{154} \mathrm{Gd}$ & ${ }^{26} \mathrm{Mg}$ & ${ }^{22} \mathrm{Ne}$ & 34 & ${ }^{94} \mathrm{Mo}$ \\
\hline 3 & ${ }^{20} \mathrm{Ne}$ & ${ }^{24} \mathrm{Mg}$ & ${ }^{156} \mathrm{Gd}$ & ${ }^{28} \mathrm{Si}$ & ${ }^{24} \mathrm{Mg}$ & 35 & ${ }^{96} \mathrm{Mo}$ \\
\hline 4 & ${ }^{22} \mathrm{Ne}$ & ${ }^{46} \mathrm{Ti}$ & ${ }^{158} \mathrm{Gd}$ & ${ }^{32} \mathrm{~S}$ & ${ }^{26} \mathrm{Mg}$ & 36 & ${ }^{96} \mathrm{Ru}$ \\
\hline 5 & ${ }^{24} \mathrm{Mg}$ & ${ }^{78} \mathrm{Kr}$ & ${ }^{160} \mathrm{Gd}$ & ${ }^{46} \mathrm{Ti}$ & ${ }^{28} \mathrm{Si}$ & 37 & ${ }^{112} \mathrm{Sn}$ \\
\hline 6 & ${ }^{26} \mathrm{Mg}$ & ${ }^{98} \mathrm{Ru}$ & ${ }^{156}$ Dy & ${ }^{48} \mathrm{Ti}$ & ${ }^{32} \mathrm{~S}$ & 38 & ${ }^{114} \mathrm{Sn}$ \\
\hline 7 & ${ }^{28} \mathrm{Si}$ & ${ }^{110} \mathrm{Pd}$ & ${ }^{158}$ Dy & ${ }^{52} \mathrm{Cr}$ & ${ }^{44} \mathrm{Ca}$ & 39 & ${ }^{120} \mathrm{Sn}$ \\
\hline 8 & ${ }^{32} \mathrm{~S}$ & ${ }^{124} \mathrm{Xe}$ & ${ }^{162} \mathrm{Dy}$ & ${ }^{56} \mathrm{Fe}$ & ${ }^{52} \mathrm{Cr}$ & 40 & ${ }^{124} \mathrm{Sn}$ \\
\hline 9 & ${ }^{34} \mathrm{~S}$ & ${ }^{130} \mathrm{Ba}$ & ${ }^{162} \mathrm{Er}$ & ${ }^{58} \mathrm{Ni}$ & ${ }^{54} \mathrm{Fe}$ & 41 & ${ }^{140} \mathrm{Ce}$ \\
\hline 10 & ${ }^{36} \mathrm{Ar}$ & ${ }^{190} \mathrm{Os}$ & ${ }^{164} \mathrm{Er}$ & ${ }^{60} \mathrm{Ni}$ & ${ }^{56} \mathrm{Fe}$ & 42 & ${ }^{142} \mathrm{Nd}$ \\
\hline 11 & ${ }^{40} \mathrm{Ar}$ & ${ }^{194} \mathrm{Pt}$ & ${ }^{166} \mathrm{Er}$ & ${ }^{64} \mathrm{Zn}$ & ${ }^{58} \mathrm{Ni}$ & 43 & ${ }^{144} \mathrm{Sm}$ \\
\hline 12 & ${ }^{44} \mathrm{Ca}$ & ${ }^{196} \mathrm{Pt}$ & ${ }^{168} \mathrm{Er}$ & ${ }^{66} \mathrm{Zn}$ & ${ }^{60} \mathrm{Ni}$ & & \\
\hline 13 & ${ }^{48} \mathrm{Ti}$ & ${ }^{198} \mathrm{Pt}$ & ${ }^{172} \mathrm{Yb}$ & ${ }^{68} \mathrm{Zn}$ & ${ }^{62} \mathrm{Ni}$ & & \\
\hline 14 & ${ }^{50} \mathrm{Ti}$ & & ${ }^{174} \mathrm{Yb}$ & ${ }^{72} \mathrm{Ge}$ & ${ }^{64} \mathrm{Ni}$ & & \\
\hline 15 & ${ }^{50} \mathrm{Cr}$ & & ${ }^{174} \mathrm{Hf}$ & ${ }^{88} \mathrm{Sr}$ & ${ }^{64} \mathrm{Zn}$ & & \\
\hline 16 & ${ }^{52} \mathrm{Cr}$ & & ${ }^{178} \mathrm{Hf}$ & ${ }^{116} \mathrm{Sn}$ & ${ }^{66} \mathrm{Zn}$ & & \\
\hline 17 & ${ }^{54} \mathrm{Fe}$ & & ${ }^{180} \mathrm{Hf}$ & ${ }^{118} \mathrm{Sn}$ & ${ }^{68} \mathrm{Zn}$ & & \\
\hline 18 & ${ }^{56} \mathrm{Fe}$ & & ${ }^{182} \mathrm{~W}$ & ${ }^{120} \mathrm{Sn}$ & ${ }^{88} \mathrm{Sr}$ & & \\
\hline 19 & ${ }^{58} \mathrm{Ni}$ & & ${ }^{184} \mathrm{~W}$ & ${ }^{124} \mathrm{Sn}$ & ${ }^{106} \mathrm{Pd}$ & & \\
\hline 20 & ${ }^{60} \mathrm{Ni}$ & & ${ }^{186} \mathrm{Os}$ & ${ }^{122} \mathrm{Te}$ & ${ }^{108} \mathrm{Pd}$ & & \\
\hline 21 & ${ }^{62} \mathrm{Ni}$ & & ${ }^{188} \mathrm{Os}$ & ${ }^{124} \mathrm{Te}$ & ${ }^{110} \mathrm{Pd}$ & & \\
\hline 22 & ${ }^{64} \mathrm{Ni}$ & & ${ }^{190} \mathrm{Os}$ & ${ }^{138} \mathrm{Ba}$ & ${ }^{110} \mathrm{Cd}$ & & \\
\hline 23 & ${ }^{64} \mathrm{Zn}$ & & ${ }^{192} \mathrm{Pt}$ & ${ }^{140} \mathrm{Ce}$ & ${ }^{114} \mathrm{Cd}$ & & \\
\hline 24 & ${ }^{66} \mathrm{Zn}$ & & ${ }^{198} \mathrm{Hg}$ & ${ }^{142} \mathrm{Nd}$ & ${ }^{116} \mathrm{Sn}$ & & \\
\hline 25 & ${ }^{68} \mathrm{Zn}$ & & ${ }^{230} \mathrm{Th}$ & ${ }^{198} \mathrm{Hg}$ & ${ }^{118} \mathrm{Sn}$ & & \\
\hline 26 & ${ }^{70} \mathrm{Zn}$ & & ${ }^{234} \mathrm{U}$ & & ${ }^{120} \mathrm{Sn}$ & & \\
\hline 27 & ${ }^{70} \mathrm{Ge}$ & & ${ }^{236} \mathrm{U}$ & & ${ }^{142} \mathrm{Ce}$ & & \\
\hline 28 & ${ }^{72} \mathrm{Ge}$ & & ${ }^{240} \mathrm{Pu}$ & & ${ }^{142} \mathrm{Nd}$ & & \\
\hline 29 & ${ }^{78} \mathrm{Kr}$ & & & & ${ }^{146} \mathrm{Nd}$ & & \\
\hline 30 & ${ }^{80} \mathrm{Kr}$ & & & & ${ }^{152} \mathrm{Sm}$ & & \\
\hline 31 & ${ }^{86} \mathrm{Sr}$ & & & & ${ }^{192} \mathrm{Os}$ & & \\
\hline 32 & ${ }^{88} \mathrm{Sr}$ & & & & ${ }^{196} \mathrm{Pt}$ & & \\
\hline
\end{tabular}




\section{References}

\section{References}

[1] J.M. Allmond, A.E. Stuchbery, A. Galindo-Uribarri et al., Phys. Rev. C 92, (2015) 041303(R).

[2] A. Jungclaus, J. Walker, J. Leske et al., Phys. Lett. B 695 (2011) 110.

[3] J.M. Cook, T. Glasmacher, A. Gade, Phys. Rev. C 73 (2006) 024315.

[4] H. Scheit, A. Gade, T. Glasmacher, T. Motobayashi, Phys. Lett. B 659 (2008) 515.

[5] B. Pritychenko, M. Birch, B. Singh, M. Horoi, At. Data Nucl. Data Tables 107 (2016) 1.

[6] S. Raman, C.W. Nestor, P. Tikkanen, At. Data Nucl. Data Tables 78 (2001) 1 .

[7] P. Royston, Appl. Stat. 44 (1995) 547.

[8] J.M. Allmond, B.A. Brown, A.E. Stuchbery et al., Phys. Rev. C 90 (2014) 034309.

[9] O. Kenn, K.-H. Speidel, R. Ernst et al., Phys. Rev. C 63 (2001) 021302.

[10] J.N. Orce, B. Crider, S. Mukhopadhyay et al., Phys. Rev. C 77 (2008) 064301.

[11] T.R. Fisher, P.D. Bond, Part. and Nucl. 6 (1973) 119.

[12] H. Ronsin, P. Beuzit, J. Delaunay et al., Nucl. Phys. A 207 (1973) 557. 